\title{
Wireless Mesh network routing protocol based on multi - routing metric
}

\author{
HUANG Xin ${ }^{1}$, BAl Jun-Hui ${ }^{2}$, AN Zhi-Hong ${ }^{3}$ \\ 1.Laboratory of Low Frequency Telecommunication Technology, The 722 Research Institute, \\ Wuhan 430205, China
}

2.Department of Software Radio Technology, The 722 Research Institute, Wuhan 430205, China

3.Department of Low Frequency Technology, The 722 Research Institute, Wuhan 430205, China

Email: 147966353@qq.com

Keywords: Node Positioning System, Cluster Analysis, Node signals Signal, Multiple Nodes Source, Sub-frame, Anti-terror Attacks

\begin{abstract}
Node positioning system can quickly determine the position of the multiple nodes. On how to improve the performance of multiple nodes localization, multiple nodes localization algorithm based on integration of subframe estimation and cluster analysis is proposed. First, a signal is divided into 8 subframes. Each subframe signal using the phase variation is calculated in response to the power control weighting function, which searches the node signal source position to obtain the maximum value of the subframe estimation. Because the signal in the time domain node is sparse, these estimates correspond to a plurality of node signals position. Then the estimated values of the sub-frames are divided into several categories using the convergence of clustering algorithm. Finally controlled in response to the sub-frame by the average estimated value of the power function was evaluated to give the final node source location estimation. Experimental results show that in the case of 2-3 nodes, the positioning performance of the algorithm is much higher than traditional algorithms.
\end{abstract}

\section{Introduction}

Microphone array system can be widely used in car hands-free communication [1], speech separation [2], the node source monitoring [3] and other fields. Node source localization based on microphone array technology has played a key role in these systems. Such as hands-free communication systems, it may use the beam source array according to the estimated orientation algorithm to suppress the formation of interference and noise. Since the 1970s, researchers at home and abroad to node positioning method conducted in-depth research, the various positioning algorithm and used the actual node signals localization system [4]. At present, a single node signal source localization based on a growing number of researchers has begun to focus on multiple nodes signal localization techniques.

\section{The proposed algorithm}

Based on the received sub-frame node signals localization techniques divided the array microphone array into subframe first. Then estimate the location of the node signal according to one or more frames. As mentioned above, the time-frequency analysis algorithm requires tens to hundreds of frame signal in order to estimate the position of the plurality of node signals. The length of frame signal is several tens of milliseconds, so this type of algorithm is used only for a stationary node signals positioning. SRP-PHAT algorithm only needs one data to estimate the location of the node signals, with a wider range of practical value.

We used $x_{m}(n)$ to represent the $m$ th microphone receives a frame data. $X_{m}(k)$ indicates its discrete Fourier transform. In the far-field assumption, the positioning algorithm only estimated arrival angle of the node signals direction (Direction of Arrival, DOA), that is node signals direction, denoted as $\boldsymbol{q}=(\theta, \phi)$. Where, $\theta$ and $\phi_{\text {respectively represent the horizontal angle and the elevation }}$ 
of the node signals. PHAT weighted pilot signals received by the array response may be expressed as:

$$
\hat{Y}^{\mathrm{PHAT}}(k, \boldsymbol{q})=\sum_{m=1}^{M} \frac{X_{m}(k)}{\left|X_{m}(k)\right|} e^{-j \omega \tau_{m l}(\boldsymbol{q})}
$$

Where $\tau_{m l}(\boldsymbol{q})$ represents the delay of $m t h$ microphone signal reaches the reference primitive $l . \omega$ represents the frequency point $k$ corresponding analog angular frequency, $M$ is the number of array primitives. The expression is:

$$
\tau_{m l}(\boldsymbol{q})=\boldsymbol{\zeta} \cdot\left(\boldsymbol{r}_{m}-\boldsymbol{r}_{l}\right) / c
$$

Where $" \cdot "$ represents the vector dot product. $\zeta$ is a representative of the node signals direction vector, its length is 1 , can be expressed as

$$
\zeta=[\cos \phi \cos \theta, \cos \phi \sin \theta, \sin \phi]^{\mathrm{T}}
$$

$\boldsymbol{r}_{m}=[x y z]^{\mathrm{T}}$ is the coordinate vectors for the $m$ th microphone in the rectangular coordinate system. ${ }^{c}$ is the air speed of node(about $342 \mathrm{~m} / \mathrm{s}$ ). Imaginary the SRP-PHAT function of node signals location $\boldsymbol{q}_{\text {for }}$

$$
\hat{P}^{\mathrm{PHAT}}(\boldsymbol{q})=\sum_{k=0}^{K-1} \hat{Y}^{\mathrm{PHAT}}(k, \boldsymbol{q}) \hat{Y}^{\mathrm{PHAT}^{*}}(k, \boldsymbol{q})
$$

Where "*" denotes taking conjugate. $K$ is the number of FFT points. According to the symmetry of the power spectrum in order to save computation, the actual calculation only need to calculate half of the frequency. The formula (4) can be written as

$$
\hat{P}^{\mathrm{PHAT}}(\boldsymbol{q})=\sum_{k=0}^{K / 2-1} \hat{Y}^{\mathrm{PHAT}}(k, \boldsymbol{q}) \hat{Y}^{\mathrm{PHAT}^{*}}(k, \boldsymbol{q})
$$

Suppose there is only one node signals, $Q$ represents the interested node signals space, then $\hat{P}^{\text {PнAT }}(\boldsymbol{q})$ reaches a maximum at the location of the real node signals, namely the location of the node signals is estimated to be

$$
\hat{\mathbf{q}}_{s}=\arg \max _{\boldsymbol{q} \in Q} \hat{P}^{\mathrm{PHAT}}(\boldsymbol{q})
$$

In the case where a plurality of node signals, each source would theoretically produce a peak, that $\hat{P}^{\text {PHAT }}(\boldsymbol{q})$ function has a plurality of peaks, each peak to identify the position of multi-node signals localization can be achieved. Literature [10] algorithm is according to the principle, which uses the converging clustering algorithm (AC: Agglomerative Clustering) to obtain the position estimate of the plurality of node signals. As mentioned above, since the mutual interference between the node signals, the peak of the node signals overlap, positioning performance of the algorithm is low.

\section{Experiment and analysis}

In order to contrast the performance of improved algorithm with the original algorithm, this research recorded a $7.62 \mathrm{~mm}$ assault rifle firing at the time of the blast wave signal as the node signals. The maximum position measurement error is taken $2 \mathrm{~cm}$ in the simulation, and the maximum delay estimation error is 5 microseconds, for pure node signals localization experiment. The microphone array for collecting signals is a radius of $1.5 \mathrm{~m}$ of uniform circular array, which is placed in a level. The number of microphones is 8, shown in Figure 1. DOA vector pointing from

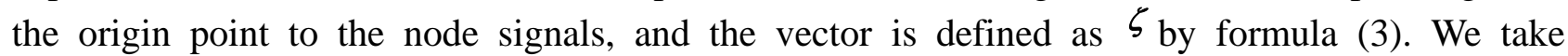
collection database Numbers "recorder-2014-04-0001" as a group of data, which has three shots. The microphone array and signals location are shown in Figure 2. 

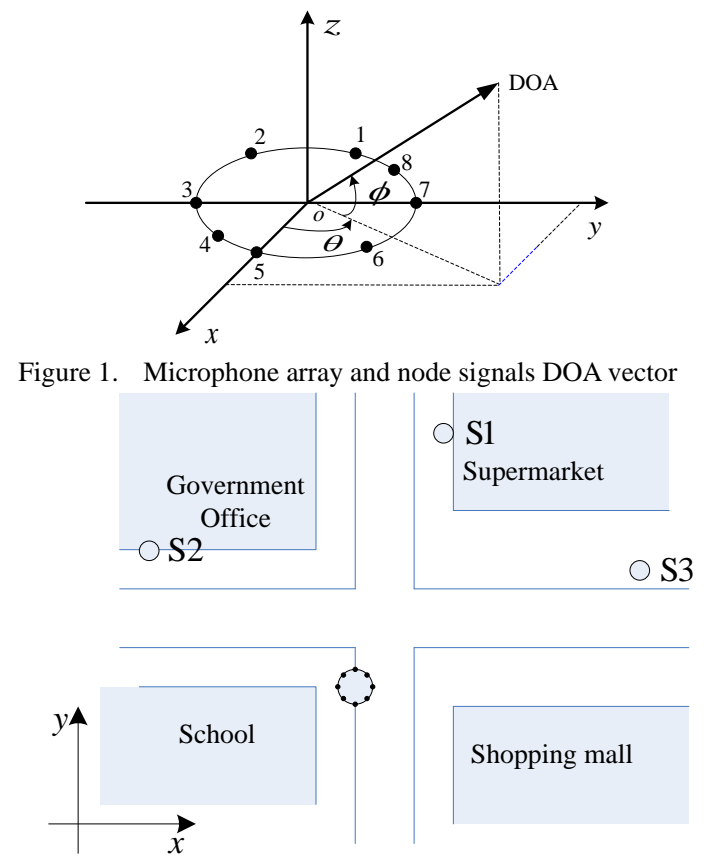

Figure 2. Microphone array and signals source location

Three signals orientation are: $\boldsymbol{q}_{s 1}=\left(74^{\circ}, 21^{\circ}\right), \boldsymbol{q}_{\mathrm{s} 2}=\left(-7^{\circ}, 24^{\circ}\right)$ and $\boldsymbol{q}_{\mathrm{s} 3}=\left(-50^{\circ}, 14^{\circ}\right)$. In the process of shooting incident, shot first single occurrence, which is s1 and s2, s1 and s3, s2 and s3, then three people shot together. In each case, we take $12.5 \mathrm{~s}$ data, a total of 50 s data. Signal sampling frequency is $16 \mathrm{kHz}$. The frame length is $T=1024$ points $(64 \mathrm{~ms})$. The number of sub-frames per frame is $N_{S F}=8$. Each sub-frame is added Henning window; the frame overlap rate is zero. After removing the silent gap, a total of 625 frames data used in the experiment, including 439 of two signals, and 186 frames of three signals.

The main purpose of the algorithm is to calculate the minimum area containing the target. First, freely choose two nodes detected the target information to calculate the intersection boundary of two circular detection areas. Then find the intersecting circular detection area between the line and $\left[T_{1}, T_{2}\right]$ through an iterative approach. After these two steps, can find the node satisfying the condition, and may calculate the straight line $d_{2}$ passing through the two points $\left\{R_{1}, R_{2}\right\}$. Then we use the same method to calculate a straight line $d_{2}$ through the circular area of ${ }^{\left[R_{1}, R_{2}\right]}$. Finally, the area obtained is the cross area of ${ }^{c_{i}},{ }^{c_{j}}$ and ${ }^{c_{k}}$, as shown in Figure 3. "+" represents the target position.

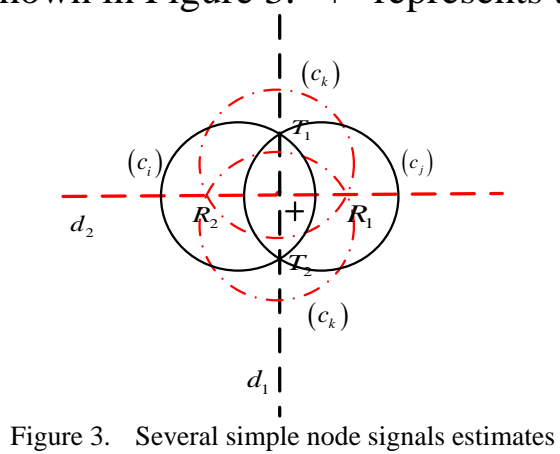

\section{Conclusion}

In the real environment, noise around is an important factor affecting the performance of node signals localization algorithm. SRP-PHAT algorithm of reverberation has stronger robustness and has been widely applied. But the spatial resolution of SRP-PHAT is low. In the case of multiple signals, the space for each node signals peaks often overlap, resulting in this algorithm has poor performance of the multiple node signals localization. This paper proposed fusion subframe 
estimate and cluster analysis of multiple node signals localization algorithm. One frame of node signal is divided into eight subframes. The subframe is calculated SRP function to search the strongest node signals position sub-frame in each sub-frame. Since the speech signal has time domain sparsely, most node signals localization estimates obtained for each subframe can not correspond to the same source, include multiple node signals location. Using AC cluster algorithm to these estimates is divided into several classes, and using the average to evaluate the estimate through function SRP, thereby obtain the final node signals position estimation. Use real environment $2 \sim 3$ node microphone array data recording experiment. The results show that the improved algorithm can effectively improve the positioning performance.

\section{References}

[1] Andrew R, Peters G P, Lennox J. Approximation and regional aggregation in multi-regional input-output analysis for national carbon footprint accounting. Economic Systems Research, 2009, 21 (3): 311-335.

[2] Boyd J P, Fitzgerald W J, Mahuta M C, Smith D A. Computing continuous core/periphery structures for social relations data with MINRES/SVD, Social Networks, 2010, 32 (2): 125-137. Kwak S J, Yoo S H, Chang J I. The role of the maritime industry in the Korean national economy: an input-output analysis. Marine Policy, 2011, 29(4): 371-383.

[3] Labaj M. Qualitative input-output analysis and national innovation system in Slovakia. International Journal of Transitions and Innovation Systems, 2011, 1 (2): 105-116.

[4] Roepke H, Adams D, Wiseman R. A new approach to the identification of industrial complexes using input-output data. Journal of Regional Science, 2000, 14 (1): 15-29

[5] Yishuang Geng, Jin Chen, Ruijun Fu, Guanqun Bao, Kaveh Pahlavan, Enlighten wearable physiological monitoring systems: On-body rf characteristics based human motion classification using a support vector machine, IEEE transactions on mobile computing, 1(1), 1-15, Apr. 2015 